In addition, the comments from the trainees suggested being 'undervalued', 'forgotten about in hospital wide conversations relating to learning/change', and 'felt junior doctors are left to get on with it'.

\section{The Network features}

- Trainee leads from each speciality

- All network members in contact via email and WhatsApp

- Periodic newsletters with opportunities at the trust, circulated by the network members

- Extra training slots were generated to facilitate trainees in existing training workshops, such as in finance and management.

- Allows for cross speciality collaborations in projects, and also a direct access for trainees to the trust leaders.

- Trainees could share other opportunities available to them, such as committee reps.

The network is growing with increasing numbers of volunteers to take up the lead roles. We hope this is a step in the right direction in finding our future leaders.

\section{LEADING INNOVATION AND IMPROVEMENT}

Maria van Hove, Anya Gopfert. Junior Doctor, Previous National Medical Director's Clinical Fellow

\subsection{6/leader-2019-FMLM.102}

Low morale among junior doctors has been attributed to a loss of the 'firm' or feeling of belong to a team as well as an increasingly demanding job, reduced resources and significant rota gaps. Improved teamwork is also associated with improved clinical outcomes. It is essential that we invest in and prioritise teambuilding for clinical staff in order to improve their wellbeing and to improve outcomes for patients. We developed an interactive, educational team-building workshop delivered in the form of an escape room, as a two-hour session including an extensive debrief and time for reflection. The Aims of the session are a) for participants to build inter-personal relationships with their colleagues b) to gain an understanding of their own role in a team. We obtained feedback from participants immediately, and nine months after the session in the form of survey data and qualitative responses. Immediate and longterm feedback were extremely positive and suggested that the training had significant improved participants' knowledge and understanding of working in a team. All participants reported that they were able to get to know their colleagues better, and that the session was enjoyable. Participants reported an improved understanding of team dynamics, a deeper awareness of their own role within a team, an increased knowledge of common team challenges and reported feeling more prepared for managing team conflict and delegating tasks. Feedback from participants after nine months particularly emphasised how the training had helped them prioritise effective communication with their team. An escape room scenario provides a novel, fun and effective way to both build a team and to improve understanding of teamworking. Participants were able to learn in a fun, innovative way, and feedback indicated that the training was successful. This is an affordable, innovative intervention which can be used for improving morale among clinical teams.

\section{KEEP THE WIRES AWAY. A QI PROJECT TO REDUCE THE OVERUSE OF CARDIAC TELEMETRY BEDS IN CAUSEWAY HOSPITAL}

Maysa Salman. Causeway Hospital, UK

10.1136/leader-2019-FMLM.103

Introduction ECG monitoring has been and remains to be an integral part of patient care in hospital. Arrhythmia detection has been reported to affect the clinical management in around $3.4-12.7 \%$ of patients. The American Heart association in collaboration with the American College of Cardiology published a statement in 2004 addressing the use of non-intensive cardiac telemetries stratifying patients into 3 different categories: cardiac telemetry is indicated, may provide benefit, or is unlikely to provide benefit. 123 telemetry beds were requested for acute admissions in October, including 13 post lysis stroke patients costing more than 30000 GBP

Methods We have adapted the AHA recommendations and formulated a local telemetry protocol in Causeway. Copies of the adapted guidelines were incorporated in the medical admission booklets, and circulated among the nurses as well as the bed managers. Junior doctors were trained about the protocol. There were 2 parts for this project. Part 1 was aimed at reducing the number of inappropriate cardiac monitors being used, while part 2 was aimed at reducing the duration in which cardiac monitors were used for.

Results Among the admissions, around 30\% of referrals were inappropriate and not indicated. Looking into the financial aspect of it, each bed costs around 300 GBP per night, leading to a total of around 30000 GBP for the total admission per night. After launching the cardiac telemetry protocol, there were a total of 89 telemetry bed requests, of which only $3 \%$ were inappropriate or not indicated, and an overall reduction of $10 \%$ in all the admissions, and $26 \%$ reduction in the inappropriate referrals. There was an overall $28 \%$ reduction of cost, saving around 10000 GBP during the first month period.

Conclusion Telemetry monitoring requires both trained personnel and specialized equipment, thus making one of the most costly equipment in the hospital setting. Guidelines remain a tool to aid but not replace clinical judgment.

\section{INCREASING THE PROPORTION OF VERBAL HANDOVERS OF ACUTELY UNWELL PATIENTS AT TORBAY HOSPITAL}

${ }^{1,2}$ Esther Oluseyi Bamigboye*,${ }^{1}$ Yuanpei Zhang, ${ }^{1}$ Clarissa Grondin. ${ }^{1}$ Torbay Hospital, UK; ${ }^{2}$ Derriford Hospital, UK

\subsection{6/leader-2019-FMLM.104}

Introduction This quality improvement project, run by three Foundation year 1 (F1) doctors, occurred at Torbay Hospital, a district general hospital in Devon England. The aim was to make the process of F1 Acute Reviews of unwell patients during weekend safer and more efficient.

Method Over a 3-week period, the number of acute reviews F1s received via text or a phone call on Saturday and Sunday was collated to ascertain the percentage of verbal acute review handover. We surveyed and spoke to nurses and members of the Hospital-at-day-team to determine their feelings on the subject. 


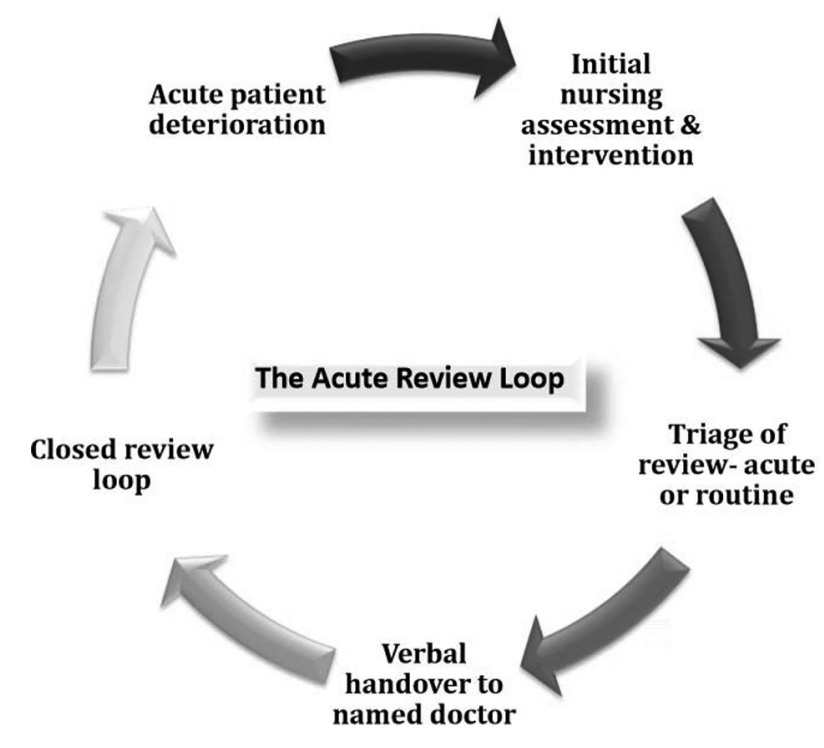

Abstract 104 Figure 1 The acute review loop

Results After analysing the preliminary data, we requested that the Hospital-at-day-team call F1 doctors with acute reviews, reverting to text only if unable to reach the F1. We trialled this intervention for 3 weeks and improved verbal handovers of acute reviews from $5.6 \%$ (1/18 verbal) to $71 \%$ (22/31 verbal). This ensured a closed acute review loop could be created in $71 \%$ of cases. We define a closed acute review loop as a system in which a named doctor is aware of an acutely unwell patient (figure 1).

Conclusions Due to our intervention, a named F1 was made aware acutely unwell patient within a short, known period, and thus could arrive in a timelier fashion ultimately improving patient care efficiency and safety. During this trial, we found that incomplete verbal SBARs were given to F1s, and routine reviews were inappropriately sent out as acute reviews.

The Out of hours taskforce at the hospital trialled a weekend of clinical staff giving advice to nurses and HCAs requesting acute reviews to facilitate the initiation of nursing level interventions at an earlier stage in the acute review process; they also gave the F1 doctor a verbal handover of acute reviews. We are working with the clinical site managers to triage reviews, ensure continuity of verbal handovers.

\section{WHY TRANS HEALTH MATTERS IN GENERAL PRACTICE}

Kamilla Kamaruddin. GP principal, East One Health Surgery,London

\subsection{6/leader-2019-FMLM.105}

I am a transgender GP and I would like to share my experience as a transgender GP and a patient to highlight the difficulties that a trans person negotiating their health care through the NHS. My journey has not been straight forward but eventually it was fulfilling and I hope my story will raise trans awareness about gender identity, improving care for trans people and inclusivity. As a trans GP, transgender issues have been close to my heart, but it seems that they have only recently risen to prominence across the society - as well as in the press, sometimes for the better, and sometimes for the worse I will present two articles which was published to highlight trans health.

1. What's it like to be a transgender patient and a GP. 313.BJGP, July 2017

2. Why Trans Care Matters. RCGP Bulletin Spring 2019

I am grateful to this country and the NHS for giving me the opportunity and protection to be what I am. I am from a vulnerable group ; a trans person, an immigrant and people of colour and I am very lucky to work in an institution like the NHS, who welcomes everyone who is willing to work hard irrespective of their gender, race and sexual orientation.

\section{DEVELOPING A HIGH QUALITY BARRETT'S OESOPHAGUS SURVEILLANCE PROGRAM OUTSIDE A TERTIARY CENTRE}

Lovesh Dyall, Georgina Chadwick. West Middlesex University Hospital, Chelsea and Westminster NHS Foundation Trust, UK

\subsection{6/leader-2019-FMLM.106}

Introduction The importance of early recognition of dysplasia within a segment of Barrett's oesophagus (BO) is well recognised, due to the risk of progression to oesophageal cancer. National guidelines on endoscopic surveillance are published in Gut 2014.

Method A retrospective audit of endoscopies for all patients with BO between January and November 2018 was conducted.

Data collected included the number of endoscopists involved, patient characteristics, and adherence to the Prague and Seattle biopsy protocol. The histology data was extracted from the pathology reporting system and follow up was checked using endoscopy reports and clinic letters.

Results 136 cases of BO were identified by 17 different endoscopists. $47 \%$ had known a diagnosis of $\mathrm{BO}$, and the remaining patients had a new diagnosis. $88 \%$ cases were reported using Prague Classification. The Seattle biopsy protocol was adhered to in $8266 \%$ cases.

Only $2 / 8$ cases of dysplasia were confirmed by a second independent pathologist. The BSG guidance states that all cases must be reviewed by a second pathologist.

The plan for follow up varied: $47 \%$ outpatient clinic, $25 \%$ virtual clinic, 5\% GP, 4\% MDT and 19\% unclear.

Conclusions This audit highlighted the need for a structured surveillance program for detection and management of dysplasia in patients with BO.

Key issues highlighted were large number of endoscopists involved and lack of a standard approach to tissue sampling, reporting and follow up. Booking of surveillance was variable and inadequate. Patients were also unnecessarily being booked into clinics- suggesting that resources were not being used the most effectively.

Results were presented at a local department meeting and the following changes agreed;

1. Introduction of regular $\mathrm{BO}$ surveillance lists by a dedicated endoscopist to standardise reporting, improve on dysplasia detection.

2. Follow up with initial clinic appointment to discuss diagnosis and surveillance, thereafter via virtual clinic. 